\title{
COVID-19 Leads to Dramatic Changes in the Food Retail Environment in New York City: May-July 2020
}

\author{
Stella S. Yi ${ }^{1}$. Shahmir H. Ali ${ }^{2}$. Rienna G. Russo ${ }^{1}$ - Victoria Foster ${ }^{1}$ - Ashley Radee ${ }^{3}$. Stella Chong ${ }^{1}$ - Felice Tsui ${ }^{4}$. \\ Julie Kranick ${ }^{1} \cdot$ David Lee $^{1,5} \cdot$ Valerie Imbruce $^{6} \cdot$ Tamar Adjoian Mezzacca $^{7}$
}

Accepted: 12 June 2021 / Published online: 13 July 2021

(c) The Author(s), under exclusive licence to Springer Science+Business Media, LLC, part of Springer Nature 2021

\begin{abstract}
A systematic assessment of the effect of COVID-19 on the food retail environment—an important determinant of health-has not been conducted. Our objective was to assess the impact of COVID-19 on closures of restaurants, food retail stores, and fresh produce vendors in New York City (NYC). We conducted a cross-sectional study following the peak of COVID-19 in six neighborhoods in NYC. Two Chinese ethnic neighborhoods and four higher/lower resourced comparison neighborhoods were selected a priori based on 14 sociodemographic indicators. The primary outcome was indefinite/temporary closures or absence of food businesses. Of 2720 food businesses identified, produce vendors and restaurants were more likely to close than food retail stores. A higher proportion of food businesses closed in Chinese ethnic neighborhoods vs. comparison neighborhoods. COVID-19 impacted food businesses in six NYC neighborhoods examined in this period, with the greatest effect observed for Chinese ethnic neighborhoods.
\end{abstract}

Keywords Food retail environment · Immigrant health · COVID-19 · New York City · Xenophobia · Chinese American · Asian American

Shahmir H. Ali and Rienna G. Russo have contributed equally to this project.

Stella S. Yi

Stella.Yi@nyulangone.org

1 Department of Population Health, NYU Grossman School of Medicine, 180 Madison Avenue, 8th Floor, Room 8-13, New York, NY 10016, USA

2 Department of Social and Behavioral Sciences, School of Global Public Health, New York University, New York, USA

3 NYU Grossman School of Medicine, New York, USA

4 Columbia Mailman School of Public Health, New York, USA

5 Department of Emergency Medicine, NYU Grossman School of Medicine, New York, USA

6 Environmental Studies Program, Binghamton University, State University of New York, Binghamton, NY, USA

7 New York, USA

\section{Background}

COVID-19 has exposed deep disparities related to structural racism and income inequality, that give rise to inequitable food access and economic opportunity. One sector that has been particularly affected is the food service industry. On March 22, 2020, New York State (NYS) on Pause went into effect and shuttered all non-essential businesses statewideincluding indoor dining at restaurants. Numerous media and reports point to the initial and ongoing struggle of restaurants and small businesses [1-4]. While also documenting an increased demand for groceries and the services of food retail stores [5]. Despite this, the cumulative effect of these factors on the food retail environment - an important determinant of health — has not been characterized.

Moreover, reports as early as January 2020 revealed the troubling effect that a new strain of coronavirus was having on businesses in Manhattan's Chinatown; tourism and business were down in part due to decreased visitors from China, but also purportedly due to fear of contracting the virus if one were to visit Chinatown [6,7]. Community partners were also reporting concern about the survival of small businesses and the well-being of neighborhood residents. 
To shed light on the economic impact faced by the NYC food retail environment during COVID-19 (including the potentially disproportionate impact faced by Chinese neighborhoods), we undertook an investigation to analyze closures of food retail stores, restaurants and produce vendors in six New York City (NYC) neighborhoods during the pandemic. We hypothesized that closures would differ across food business type, and further, given COVID-related xenophobia, that businesses in Chinese ethnic neighborhoods would be particularly affected.

\section{Methods}

The current study did not include human subjects research and was granted exemption from the New York University Grossman School of Medicine Institutional Review Board.

\section{Neighborhood Selection}

Data from this work is a part of the COVID Closures (CoClo) project, which was launched in April 2020 to understand the interrelated issues of food access, economic security and racism. The Coclo Project includes both quantitative and qualitative data collection and analysis, as well as simulation modeling to assess these impacts. Herein we describe results from the quantitative data collection activities. For this component we utilized administrative data and ground truthing to enumerate changes to the food retail environment in select NYC neighborhoods. We defined neighborhoods as neighborhood tabulation areas (NTAs). NTAs were developed by the NYC Department of City Planning, each of which has a minimum population of approximately 15,000 and were created using whole census tracts in order to align with other geographic projects such as the Public Use Microdata Areas (PUMAs) [8]. We opted to focus on these smaller geographic areas In order to make the data collection feasible within the timeframe and with available person power on our team. The neighborhood selection process was as follows: first the two most populous Chinese ethnic neighborhoods were selected: Manhattan Chinatown and Sunset Park in Brooklyn. To select comparison neighborhoods, we relied on data from the NYC Neighborhood Health Atlas which reports on numerous sociodemographic and health factors for 59 community districts in NYC. Community districts are larger geographic areas that encompass NTAs with similar but not precisely equivalent boundaries as NTA neighborhoods [9]. We examined all community districts in Manhattan and in Brooklyn on 14 different factors including food access (supermarket: bodega ratio), demographics (population, foreign-born, limited English proficiency), socioeconomic factors (poverty, unemployment, rent burden, uninsured), health behaviors (physical activity, fruit \& vegetable (FV) and sugary drink consumption) and chronic conditions (obesity, diabetes, hypertension). We then selected the higher and lower resourced comparison neighborhoods in Manhattan and Brooklyn. There were no universal best/worst neighborhoods on all characteristics; thus final selection was reached through group discussion and with longtime NYC residents. The higher/lower resourced neighborhoods selected were the Upper East Side (UES) and East Harlem in Manhattan; and Park Slope and Brownsville in Brooklyn. Select characteristics pulled from the NYC Department of Health and Mental Hygiene Community.

Health Profiles are available in Supplemental Table S1.

\section{Building Complete Lists of Food Retail Stores, Restaurants and Grocery Stores}

CoClo methods are described elsewhere 10,11]. In brief, food retail stores and restaurants for each of the six neighborhoods were ascertained by the most up-to-date NYS Agriculture and Markets Retails Stores list and by the NYC Health Department Restaurant Inspections database (last updated March 16, 2020). Existing locations of produce vendors were available for Chinatown [11, 12] and verified using Google Street View (last neighborhood update, June-October 2019). Locations of produce vendors were not available in other neighborhoods but were ascertained using Google Street View to conduct virtual walks down each street of each neighborhood [11]. Because NTA information was not available in either the NYS or NYC Health Department lists, ArcGIS was used to map out all the restaurants and food retail stores, and then identify which stores were within the boundaries of each of our six study neighborhoods.

\section{Assessing pre-COVID ‘Open' Status and During-COVID Closure Status}

Working from this 'universe' of food retail stores, restaurants and produce vendors in each of the six neighborhoods, the following process was undertaken for each neighborhood: (1) verification that location was open pre-COVID (late 2019/early 2020); (2) Internet checks by two sources (e.g., business' site, Google Maps/reviews, Yelp, etc.) of status; (3) call checks (in language-Mandarin Chinese, Spanish, as needed); and (4) in-person checks. For Step 1, there were a number of ways food businesses were verified as open pre-COVID. For example, the business might have received customer reviews on Yelp, or images were found that included calendar dates of the food retailer on Google Street View showing the business was open and active. In this step we attempted to find evidence of having been open as close to March 2020 as possible. For produce vendors, Google Street View images were used to identify 
their presence on sidewalks; the last update of the Google Street View images in the six neighborhoods were from June to Oct 2019.

The next step was to assess the status of each food retail store or restaurant as being open or closed through a threestep verification process (Steps 2-4). First, multiple internet sources were checked to find evidence that indicated whether the food retailer was open or closed from May 2020 onwards. If the food retailer was open, data on any changes to the services of the vendor was collected (e.g., a restaurant was only offering delivery). If closed, information indicating if the food retailer was temporarily closed or indefinitely closed was sought. Various sources were used to find this information, including Google maps, delivery apps (e.g., Grubhub, Chowbus), and other social media; two different internet sources were required for this verification step. If the status of the food retailer could not be verified using online sources, the next step was to directly call the food retailer and inquiring in English, Mandarin, Cantonese or Spanish, as needed. After calling the phone number at least two times, if status could not be verified, an in-person check of the food retailer was conducted. During in-person checks, a shared Google Maps account was used to note the status of the business with detailed notes. For example, the business could be open; open but operating with limited service (e.g., a visibly limited selection of produce/products on display); closed with a paper sign with additional information (English, Chinese); closed without additional information; and closed down (e.g., restaurant was empty of chairs/tables). For produce vendors, since internet information or phone numbers for the vendors did not exist, in-person checks were used to indicate presence, absence, or any changes to services (e.g., evidence of scaling-down or limiting certain services). A variable was then created representing whether the food outlet was open (opened/re-opened between June 1 and July 31), was temporarily closed (closed w/notice of reopening schedule), or an indefinite closure (closed w/no info on re-opening). Duplicates, outlets closed in 2019, anomalous locations (e.g., wedding/sporting event venues) and outlets not found were excluded. Differences in COVID-related changes were tested using F-tests and post-hoc Bonferroni test for pairwise comparisons in STATA v.12.1.

\section{Timeline}

Key dates salient to the opening and closures of food retail stores, restaurants and produce vendors-including dates of social unrest-are depicted in Fig. 1. Data collection was conducted from May 1 to July 31, 2020. Data steps were conducted in sequence (e.g., internet checks for neighborhoods at one point in time vs. data collection steps for Chinatown, then UES). Reports from community partners that documented the openings and closures of grocery stores and restaurants were reviewed and administrative data were pulled in May 2020. During this time period was the murder of George Floyd, and as a result both peaceful protesting and looting and vandalism erupted in NYC. These events bear mentioning since the Manhattan Chinatown neighborhood was geographically centered where much of this activity was occurring in the downtown area-and may have also affected the closures observed. Data collection for Manhattan occurred from June 3 rd to July 5 th, and for Brooklyn from June 18th to July 31 st during which time outdoor dining was introduced; indoor dining was postponed indefinitely, and NYC introduced Open Streets for dining including designated areas in Chinatown.

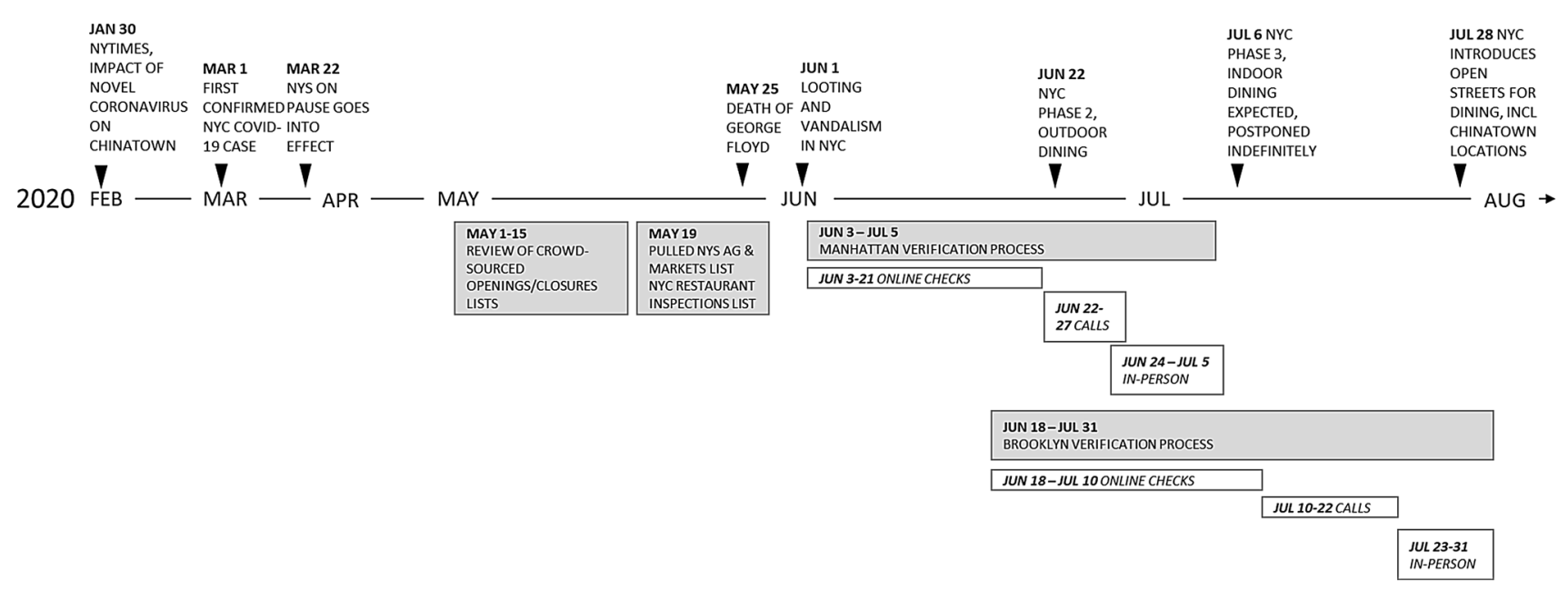

Fig. 1 Timeline of events and data collection, CoClo May-July 2020 displays the timeline of salient events pertaining to closures within the food retail environment in New York City prior to and concurrent with data collection 


\section{Results}

The six selected neighborhoods differed on the examined food access and sociodemographic factors selected (Supplemental Table S1). The lower vs. higher resourced neighborhoods had higher estimates of poverty; unemployment; rent burden; lack of health insurance; sugary drink consumption; and obesity, diabetes and hypertension. The Chinese ethnic neighborhoods had the highest estimates having populations who were born outside the U.S.; were limited English proficient; had similar or higher estimates of poverty and rent burden vs. lower resourced neighborhoods; and were 'midway' between lower and higher resourced neighborhoods for unemployment, obesity, diabetes and hypertension. April 1, 2020 - the first day for which neighborhood-level data on COVID-19 cases were available there were 4248.0 cases per 10,000 in Chinatown, 4341.3 cases per 10,000 in East Harlem, and 3142.9 cases per 10,000 in the UES, as well as 5666.7 cases per 10,000 in Sunset Park East, 5874.7 cases per 10,000 in Brownsville, and 4073.2 per 10,000 in Park Slope [13].
Of the 2720 food businesses identified (753 food retail stores, 1832 restaurants, 135 produce vendors), there were $874(32 \%)$ in Chinatown, 358(13\%) in the UES, 232(9\%) in East Harlem, 537(20\%) in Sunset Park, 512(19\%) in Park Slope, and 207(8\%) in Brownsville. The composition of food businesses was different across the six neighborhoods (Figure S1). Produce vendors were the most likely food business type to close; followed by restaurants and grocery stores (Figs. 2; Supplemental Table S2). For example, during our observation period, in Chinatown, $52 \%$ of produce vendors, $26 \%$ of restaurants and $12 \%$ of food retail stores were closed, with similar patterns observed across the other five neighborhoods.

A higher proportion of food businesses closed in Chinatown during the observation period vs. East Harlem (27\% vs. $9 \%, \mathrm{p}<0.01$; Fig. 3). Similarly, a higher proportion of food businesses closed in Sunset Park as a result of COVID-19 vs. Brownsville ( $16 \%$ vs. $6 \%, \mathrm{p}=0.01)$ and Park Slope $(16 \%$ vs. $10 \%, \mathrm{p}=0.05)$. The proportion of food businesses closed was the highest in Chinatown compared to all other neighborhoods, excluding the UES ( $\mathrm{p}<0.01$ for all vs. Brooklyn neighborhoods; two Manhattan neighborhoods differences listed above). Lastly the greatest number of food businesses

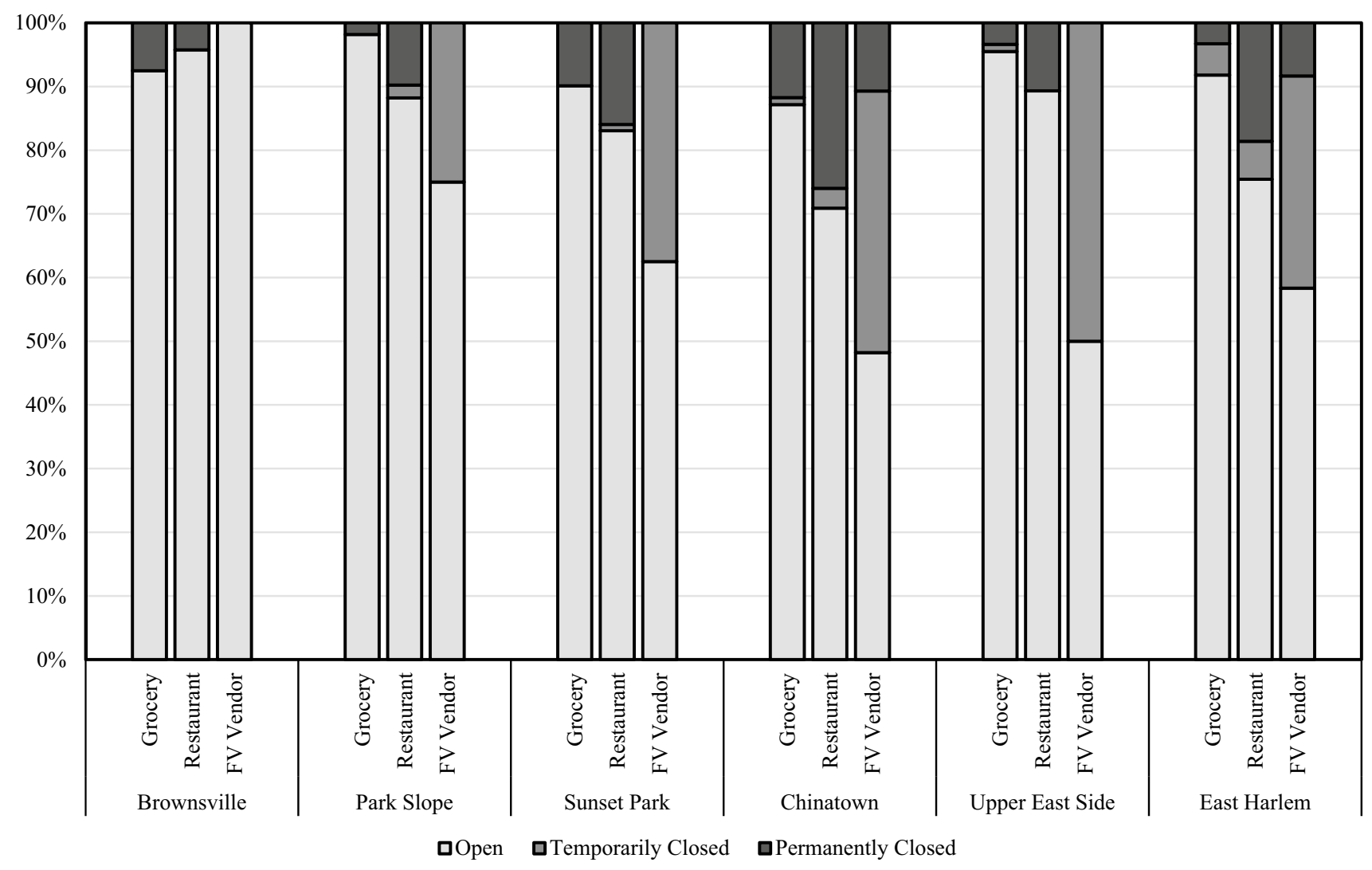

Fig. 2 Percentage of closures in restaurants, food retail stores and fruit and vegetable vendors, CoClo May-July 2020. Displays the of a restaurants; b food retail stores; and $\mathbf{c}$ fresh produce vendors were that open, temporarily closed or indefinitely closed during this time period. For fresh produce vendors, the categories were present, limited service, or absent. Actual values available in Supplemental Table S2. 


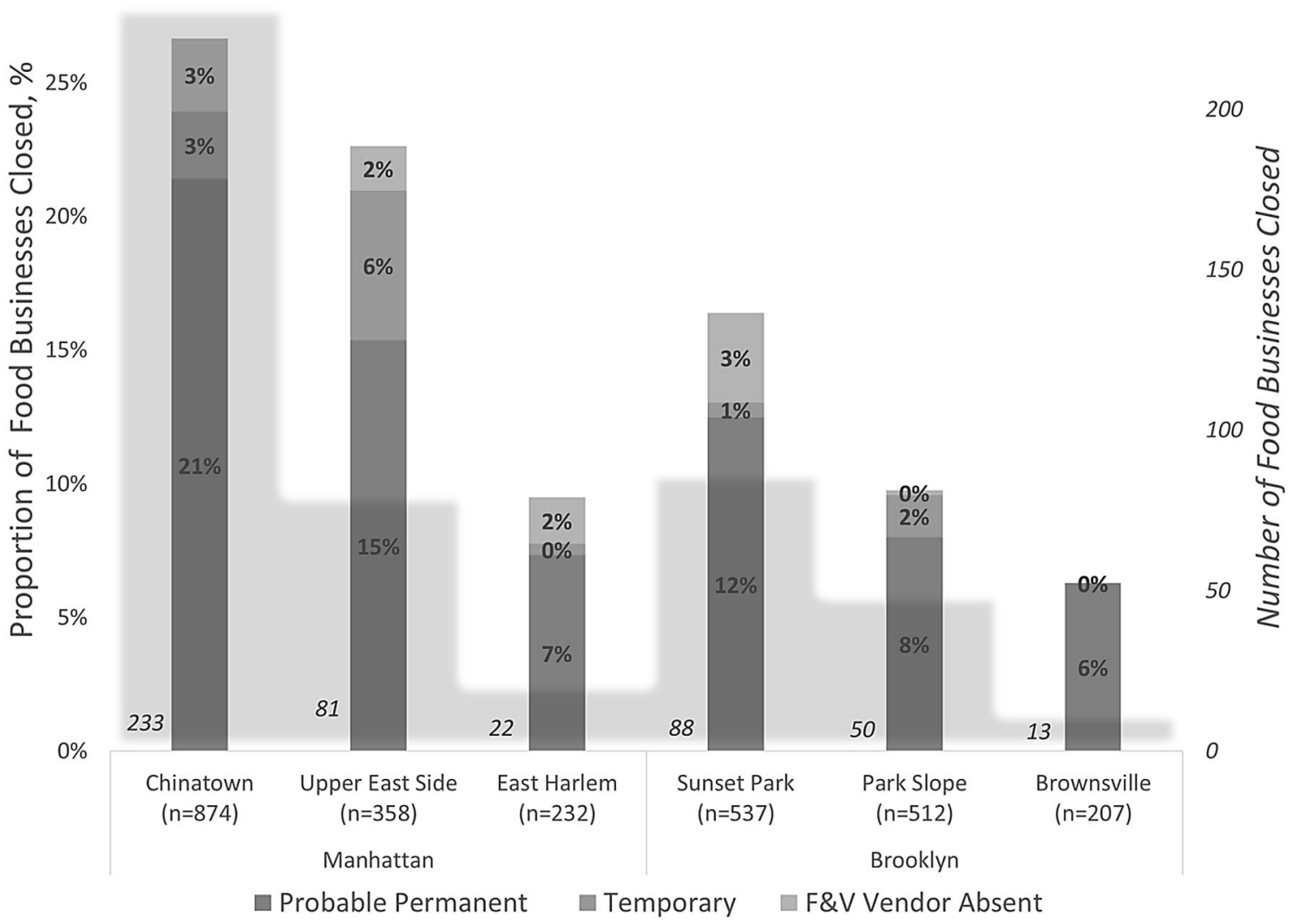

Fig. 3 Proportion and number of closures in NYC food businesses, Coclo May-July 2020. Displays the proportion of food businesses overall (restaurants, grocery stores, fruit and vegetable vendors) that

closed in Chinatown $(n=233)$ as a result of COVID-19 compared to the other five neighborhoods. A higher proportion of restaurants, food retail stores and produce vendors either closer or were absent in the Chinese ethnic neighborhoods vs. comparison neighborhoods in both Manhattan and Brooklyn (Fig. 2).

\section{Discussion}

To the authors' knowledge, this is the first investigation of the impact of COVID-19 on the food retail environment. During the observation period, COVID-19 had a large effect on businesses in the six NYC neighborhoods examined, with the greatest effect being observed for Chinese ethnic neighborhoods. Although the burden of COVID-19 in Chinatown is similar to that of the UES, there were more closures in Chinatown. These results reflect the impact of multiple effects on Chinatown including reduced tourism, COVID-19 stigma and discrimination, reduced business lunch/foot traffic, and potential fear on the part of small business owners as were closed or absent during this time period. On the tertiary axis, the \# of affected businesses are displayed

a result of nearby protests that sometimes erupted in vandalism and violence $[6,7,14,15]$. It is important to underscore the temporal nature of these results; we feel they are reflective of what was happening at this point in time.

Manhattan's Chinatown, one of the oldest and most populous Chinatowns in the country, has long been a food and tourist destination. Moreover, Chinatown serves as an important hub and source of cultural food items and produce not only for its own neighborhood residents, but for many immigrant groups within NYC and more broadly up and down the Northeast Corridor for food retail stores and restaurants. COVID-19 therefore may have farther reaching effects on food systems across the U.S. that rely on the Chinatown infrastructure to survive [16]. A report released concurrently with the timing of the CoClo data collection efforts highlights the disproportionate representation of Asian Americans in the food service industry and the economic hardship that they are newly facing [17]. indicating both deep structural and individual-level implications of this work. Moreover, in more recent months-the longer term impacts are beginning to materialize, with the highest 
unemployment rates being observed in the Asian American community, and a slower economic recovery of Asian American owned small businesses [18, 19].

A key strength of this study is the multi-tiered open verification approach to assess vendor status. Key limitations include, first, data were collected at a discrete point in time. Due to logistical limitations (e.g. call and in-person checks), a 5-week cross-sectional timeframe was used to assess vendor status; some that were closed at the start of that timeframe may have re-opened by the end. However, at the time of the study, given the indefinite delay of reopening for indoor dining in NYS [20] many small businesses were reportedly closing anyway [21]. This may be particularly true for densely populated neighborhoods like Chinatown that have fewer opportunities to allow for outdoor dining. In currently ongoing research we are conducting a second timepoint of data collection, particularly given the broader introduction of Open Streets for outdoor dining in NYC and in Chinatown specifically; the election and continued anti-China rhetoric from President Trump; and the potential second wave of COVID-19 in NYC. Second, although the administrative datasets used to identify the restaurant and food retail store were comprehensive, some vendors may have been missed in the assessment; however it is unlikely that any missingness is differential or related to neighborhood. Moreover, analysis was also limited by the number of food vendors that existed in each neighborhood, and as a result there were some significant disparities between neighborhoods (e.g., Park Slope had nearly eight times as many grocery stores as Upper East Side). Additionally, we conducted bivariate analyses and did not adjust for any significant differences in neighborhood characteristics. We acknowledge that there were differences in baseline sociodemographic characteristics of the neighborhood, as well as food retail composition. However, the purpose of this paper was to describe the preliminary findings from the study, future analyses of CoClo data should explore differences in closures after accounting for baseline differences in neighborhood characteritsics Lastly, we did not have information on a typical 'endemic' rate of closures that are typical within any given calendar year.

Construction of the CoClo dataset highlights innovative data methods that may be replicated by others looking to understand the impact of COVID-19 on the local food retail environment. The majority of this data collection was conducted virtually, maintaining social distancing and shelter in place orders. Our results have implications for food access, food systems and economic opportunity not just for, but primarily for Asian American communities.

Our results paired with emerging work support early evidence of the far-reaching and deeply embedded ways xenophobia is affecting the Asian American community if these proportions of closures are indeed permanent. While we are unable to parse out the differential impacts of being a tourist destination vs. a business center vs. xenophobia on the effects observed in Manhattan Chinatown, a few pieces of evidence point towards xenophobia being at least some part of the explanation for these results. First, as of April 1, 2020 - the first day for which neighborhood-level data on COVID-19 cases were available-there were 4248.0 cases per 10,000 in Chinatown, 4341.3 cases per 10,000 in East Harlem, and 3142.9 cases per 10,000 in the UES, as well as 5666.7 cases per 10,000 in Sunset Park East, 5874.7 cases per 10,000 in Brownsville, and 4073.2 per 10,000 in Park Slope [13]. In other words, the case counts were actually lower in the Chinese ethnic neighborhoods vs. the comparison neighborhoods. Second, Sunset Park is not a tourist destination nor proximal to a business center, yet closures were disproportionately observed there compared to comparison neighborhoods in Brooklyn. Third, Asian American owned businesses actually recovered faster thant other group owned businesses following the Great Recession [22]. Similar patterns may not hold true with the COVID-19 pandemic though it remains to be seen in the longer term [19, 23]. Lastly, the impact on Chinatowns across the nation and globally has also emerged [24-27]. Indeed, despite the fact that the source of COVID-19 for NYC was not from China but from Europe [28], xenophobia and fears of 'yellow peril' may persist long after the pandemic has passed [29, 30].

Supplementary Information The online version contains supplementary material available at https://doi.org/10.1007/s10903-021-01230-7.

Acknowledgements We'd like to extend our warmest gratitude to Samuel H. Kaplan and Kaye Stevenson - our two enthusiastic high school interns - for their invaluable assistance and insights contributing to this project. We also thank Dr. Joel Gittelsohn for his helpful input on the manuscript draft.

Funding This publication is supported by grant numbers U54MD000538 from the National Institutes of Health (NIH) National Institute on Minority Health and Health Disparities, and R01HL141427 from the National Heart, Lung and Blood Institute. The contents of this publication are solely the responsibility of the authors and do not necessarily represent the official views of the NIH.

\section{Declarations}

Conflict of interest The authors declare that they have no conflict of interest.

\section{References}

1. Carlson J. NYC Restaurants are Closing Amidst Coronavirus Pandemic. Gothamist https://gothamist.com/food/nyc-restaurants-areclosing-amidst-coronavirus-pandemic. Accessed 14 Mar 2020

2. New York State Restaurant Association. State restaurant association survey: nearly two-thirds of New York's restaurants likely to 
close by year's end without govnernment support. 2020. https:// www.nysra.org/uploads/1/2/1/3/121352550/state_restaurant_ association_survey_results_090320.pdf. Accessed 3 Sep 2020

3. New York City Comptroller, Scott M. Stringer. Save main street: a crash program to help save NYC small businesses.2020. https:// comptroller.nyc.gov/reports/save-main-street/. Accessed 5 Aug 2020

4. Office of the New York State Comptroller. The restaurant industry in New York City: tracking the recovery. 2020. https://www.osc. state.ny.us/files/reports/osdc/pdf/nyc-restaurant-industry-final.pdf. Accessed Sep 2020

5. Bogost I. The supermarket after the pandemic. The Atlantic 2020. https://www.theatlantic.com/technology/archive/2020/04/howyoull-shop-for-groceries-after-the-pandemic/610135/. Accessed 17 Apr 2020

6. Salcedo A. Coronavirus, a fire and anxiety in the Chinese community. The New York Times. Jan 302020

7. Barron J. Coronavirus in N.Y.: Without Chinese tourists, business sags. The New York Times. Feb 42020

8. NYC Department of City Planning. Neighborhood tabulation areas. 2021. https://www1.nyc.gov/site/planning/data-maps/opendata/dwn-nynta.page. Accessed 19 Feb 2021

9. New York City Neighborhood Health Atlas. 2020. https://public.tableau.com/profile/nyc.health\#!/vizhome/NewYorkCityNeig hborhoodHealthAtlas/NeighborhoodData. Accessed 15 Mar 2020

10. Russo RG, Ali SH, Mezzacca TA, et al. Methods for characterizing changes to the food retail environment during the COVID-19 pandemic. BMC Med Res Methodol. Under review

11. SH Ali VM Imbruce RG Russo 2021 Evaluating closures of fresh fruit and vegetable vendors during the COVID-19 pandemic: methodology and preliminary results using omnidirectional street view imagery JMIR Form Res 52 e23870

12. V Imbruce 2016 From farm to canal street Cornell University Press New York

13. NYC Department of Health and Mental Hygiene. COVID-19 cases. 2020. https://github.com/nychealth/coronavirus-data/tree/ c2539dc70437a42575f3c3ea8b230047de23dff3. Accessed 24 July 2020

14. Haag M. Manhattan faces a reckoning if working from home becomes the norm. The New York Times May 122020

15. N.Y.C. Protests Turn Violent. The New York Times May 312020

16. Offenhartz J. Here's why your local Chinese takeout spot is (Probably) closed. April 132020

17. Mar D, Ong P. University of Califonia Los Angeles. Asian American studies center. COVID-19's employment disruptions to Asian Americans. 2020. http://www.aasc.ucla.edu/resources/policyrepo rts/COVID19_Employment_CNK-AASC_072020.pdf. Accessed 28 July 2020

18. Khan R, Shih H. Asian American Federation. Impact of COVID19 on Asian American employment in New York City. Asian Americans face record job losses. 2020
19. Kuang J, Delawala I, Yang A. Community left behind: Asian American businesses affected by COVID-19. https://abcnews.go. com/US/community-left-asian-american-businesses-affectedcovid-19/story?id=72935477. Accessed 10 Sep 2020

20. McKinley JF-S, Luis. N.Y. Officials Halt Indoor Dining, Alarmed by Virus Rise in Other States. The New York Times 2020

21. Flitter E. 'I Can't Keep Doing This:' Small-Business Owners Are Giving Up. The New York Times July 132020

22. Asian American Federation. NYC's economic engine: contributions and challenges of Asian American small businesses. http:// www.aafny.org/doc/AAF_small_biz.pdf. 2016.

23. Asian American Advocacy Fund. Asian-owned small businesses \& COVID-19. 2020. https://www.asianamericanadvocacyfund. org/asian-owned-small-businesses-covid-19. Accessed 18 Feb 2021

24. Foster A. 'Slashed business in half': Chinatown deserted over virus fears. news.com.au Web site. 2020. https://www.news.com. au/finance/business/slashed-business-in-half-chinatown-desertedover-virus-fears/news-story/d27d58a1b55d7c73415661102b5d68 d9. Accessed 18 Feb 2020

25. MacKenzie A, Kovac A. COVID-related racism, lack of tourists combine to devastate Montreal's Chinatown. 2020. https://montr eal.ctvnews.ca/covid-related-racism-lack-of-tourists-combineto-devastate-montreal-s-chinatown-1.5115988. Accessed 18 Feb 2021

26. Coronavirus: Fears over London Chinatown's survival. 2020. https://www.bbc.com/news/uk-england-london-52605319. Accessed 18 Feb 2021

27. Gee L. San Francisco's Chinatown clings to hope amid COVID-19 pandemic. 2021. https://abc7news.com/san-francisco-chinatowncoronavirus-sf-covid-merchants-association/9735798/. Accessed 18 Feb 2021

28. D Bushman KA Alroy SK Greene 2020 Detection and genetic characterization of community-based SARS-CoV-2 infectionsNew York City, March 2020 MMWR Morb Mortal Wkly Rep 69 28918922

29. Borja M, Jeung R, Horse AY, et al. Anti-Chinese rhetoric tied to racism against Asian Americans stop AAPI hate report. June 22, 2020.

30. Silver L, Devlin K, Huang C. Americans fault China for its role in the spread of COVID-19. Pew Research Center. Global Attitudes and Trends July 30, 2020.

Publisher's Note Springer Nature remains neutral with regard to jurisdictional claims in published maps and institutional affiliations. 